\title{
FACTORES PRONÓSTICOS ASOCIADOS A MALA EVOLUCIÓN EN PACIENTES OPERADOS DE FRACTURA DE CADERA MAYORES DE 65 AÑOS
}

\author{
PRONOSTIC FACTORS ASSOCIATED WITH BAD EVOLUTION IN PATIENT OPERATED HIP \\ FRACTURE OVER 65 YEARSOLD
}

Renee Flor Vento-Benel ${ }^{1, a}$, Cecilia Salinas-Salas ${ }^{1, b}$, Jhony A. De la Cruz-Vargas ${ }^{1, c, d, e}$

\begin{abstract}
RESUMEN
Introducción: La fractura de cadera en el adulto mayor es la causa más común de hospitalización en el servicio de traumatología, ocasionando repercusión en distintas áreas clínicas y afectando su independencia. Objetivo: Determinar los factores pronósticos de mala evolución, en la población de estudio. Métodos: Se diseñó un estudio ambispectivo, correlacional y no experimental. La población estuvo constituida por todos los pacientes mayores de 65 años intervenidos quirúrgicamente por fractura de cadera, entre enero de 2014 hasta julio 2015 del Hospital Central de la Fuerza Aérea del Perú (F.A.P). Se cuantificó la magnitud de la asociación utilizando el odds ratio, posteriormente se hizo un análisis multivariado para las variables significativas. Resultados: Las variables identificadas con un fuerte grado de asociación a mala evolución (grado funcional desfavorable) a los 6 meses de seguimiento fueron: edad mayor de 75 años (OR=3,750 IC: 1,275-11.026 p=0,016), alta comorbilidad (OR=4,00 IC: 1,58$10.08 p=0,003$ ), hemoglobina < 10 (OR 6,545 IC: 1,17-36.6 $p=0,0032$ ), complicación respiratoria (OR 6,00 IC: $1,55-22.94 p=0,000$ ). La fractura previa mostro ser factor pronostico asociado a mortalidad (OR 3,665 IC: 1,366- 9.821 p=0,010). Conclusión: La mala evolución en fractura de cadera está asociada a una elevada comorbilidad, edad mayor de 75 años, residencia, complicación respiratoria postoperatoria con importantes limitaciones en la capacidad de la marcha y el estado funcional de los pacientes. Finalmente, el antecedente de fractura previa mostró alta asociación a mayor mortalidad de los pacientes a los 6 meses de seguimiento.
\end{abstract}

Palabras clave: Fractura de cadera; Factores pronósticos. (fuente: DeCS BIREME)

\begin{abstract}
Introduction: Hip fracture in the elderly is the most common cause of hospitalization in the trauma department, causing repercussion in several clinical areas, affecting its independence. Objective: To determine the prognostic factors of poor evolution in the study population. Methods: Ambispective, correlational and non-experimental study was designed. The population consisted of all patients over 65 years of age who underwent surgery for hip fracture between January 2014 and July 2015 at the Central Hospital of the Pruvian Air Force (F.A.P). The magnitude of the association was quantified using the odds ratio, and a multivariate analysis was performed on the significant variables and a 6-month follow-up was performed. Results: The variables identified with a strong association with poor evolution were: age greater than 75 years $(\mathrm{OR}=3,750 \mathrm{Cl}: 1,275-11,026 \mathrm{p}=0,016)$, high comorbidity $(\mathrm{OR}=4.00 \mathrm{Cl}: 1.58-10.08 \mathrm{p}=$ 0.003 ), hemoglobin $<10$ (OR 6,545 Cl:1.17-36.6 $p=0.0032$ ), respiratory complication (OR 6.00 Cl: $1.55-22.94$ $p=0.000$ ) patients in the 6-month follow-up. The previous fracture was strongly related to mortality (OR $3,665 \mathrm{Cl}: 1,366-9,821 p=0,010)$. Conclusions: The poor evolution in hip fracture is associated with high comorbidity, age greater than 75 years, residence (institutionalized patient), postoperative respiratory complication with significant limitations in gait capacity and the functional status of the patients. Finally, the antecedent previous fracture showed high association with an increased mortality of patients at 6 months follow-up.
\end{abstract}

Key words: Hip fracture; Prognostic factors. (source: MeSH NLM)

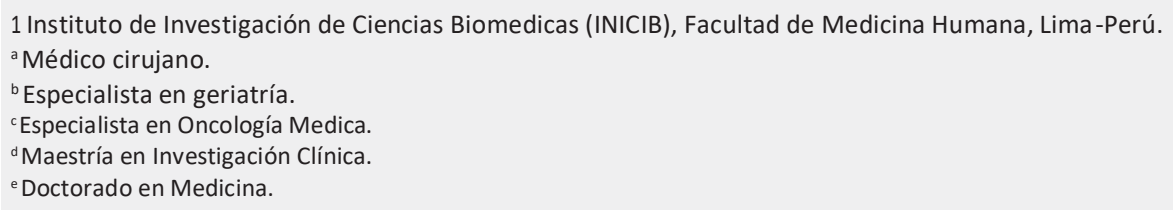

Citar como: Renee Flor Vento-Benel, Jhony A. De la Cruz-Vargas, Cecilia Salinas Salas. Factores pronósticos asociados a mala evolución en pacientes operados de fractura de cadera mayores de 65 años. Rev. Fac. Med. Hum. Octubre 2019;19(4):84-94. DOI 10.25176/RFMH.v19i4.2344 


\section{INTRODUCCIÓN}

La fractura de cadera es una entidad prevalente en el grupo etario de edad avanzada, y es una de las causas más frecuentes de ingreso hospitalario en personas mayores de 65 años; debido a que la población de adultos mayores ha ido creciendo en las últimas décadas, esta patología ha adquirido una gran importancia. Cada año ocurren alrededor de 1.5 millones de fracturas de caderal. La Organización Mundial de Salud ha estimado que para el año 2050 un total de 6 millones de fracturas de cadera ocurrirán en el mundo entero por año y en la Comunidad Europea será superior a 1 millón de fracturas2. En los Estados Unidos, el número total de fracturas de cadera en personas de 50 años o más pasará de 238.000 a 512.000 enelaño 2040, con un aumento concomitante de muertes evitables, discapacidad y costos médicos3.

En Perú y en Latinoamérica no se cuenta con cifras exactas, relacionadas a esta patología, sin embargo, si estima que para el año 2050 se incrementará este tipo de lesiones a nivel mundial y un $70 \%$ corresponderá a Latinoamérica4 y en el Perú, EsSalud (el prestador de seguro nacional de salud para los trabajadores públicos) calcula queel $12-16 \%$ delas mujeres peruanas mayores de 50 años sufrirá una fractura de cadera por año. Las estadísticas poblacionales proyectan que habrá 7,5 millones de mujeres peruanas de 50 años o más en el año 2050. Por lo tanto, se podrían esperar más de 500.000 fracturas de cadera en el año 20505, por ello algunos autores la han denominado la epidemia silenciosa en Perú6.

La mortalidad intrahospitalaria oscila entre el 4 y el $8 \%$ y se considera que alrededor de un $40-50 \%$ de los pacientes recupera la funcionalidad previa a la fractura, aunque solo un $30 \%$ volverán a ser independientes para las actividades de la vida diaria7. En estudios realizados en EE. UU se encontró que globalmente cerca del $30 \%$ de los pacientes operados por fractura de cadera fallecerán8. De hecho, la fractura de cadera es capaz por sí sola de disminuir la esperanza de vida en casi dos años y uno de cada cinco pacientes que la sufre requerirá asistencia socio-sanitaria permanente9.

Se han descrito múltiples factores que tienen influencia en la morbimortalidad perioperatoria. Algunos propios del paciente (edad, sexo, falta de autonomía, comorbilidad, demencia, polifarmacia, anemia, tipo de fractura, lugar de residencia propia o asilo geriátrico, etc. ) $^{10}$, otros intrahospitalarios (demora quirúrgica, tipo de cirugía, tratamiento anestésico, inicio de la deambulación y rehabilitación, etc. $)^{11}$ y relacionados con el alta del paciente (rehabilitación, soporte, prevención de caídas, tratamiento de la osteoporosis, etc. $)^{12}$.
Para disminuir la estancia hospitalaria y la morbimortalidad postoperatoria, es necesario optimizar el estado de salud previo a la cirugía, prevenir las complicaciones y ofrecer durante todo el proceso, de manera oportuna el tratamiento hospitalario más adecuado $^{11}$.

Por lo ya mencionado, la fractura de cadera supone un problema de gran importancia tanto para el área asistencialcomoparalaadecuadagestión hospitalaria.

El objetivo principal de este estudio fue determinar los factores pronósticos asociados a mala evolución (grado funcional desfavorable) en pacientes mayores de 65 años con fractura de cadera intervenida, e identificar los factores pronósticos asociados a la mortalidad postoperatoria a los 6 meses posteriores a la cirugía.

\section{MÉTODOS}

Se realizó un estudio de tipo ambispectivo, correlacional, no experimental, con un muestreo censal, donde fueron incluidos 99 pacientes mayores de 65 años intervenidos quirúrgicamente por fractura de cadera, entre enero de 2014 hasta julio 2015, en el Hospital Central de la Fuerza Aérea del Perú.

Se excluyeron a aquellos pacientes con diagnostico fractura patológica y a aquellos que no fueron tributarios de tratamiento quirúrgico.

Para la identificación de la población se consultó los registros hospitalarios del Servicio de Traumatología y Ortopedia. La información sociodemográfica, biológica, clínica y terapéutica se obtuvo de los registros en la historia clínica.

Se realizó un seguimiento de los pacientes a los 6 meses del alta hospitalaria, mediante las atenciones por consultorio externo y visita domiciliaria de geriatría. Las variables de estudio se agruparon en: datos sociodemográficos, biológicos, clínicos, de la fractura y su tratamiento, complicaciones postoperatorias y presencia de transfusiones sanguíneas. Además, se evaluaron parámetros funcionales y de mortalidad a los seis meses después de la cirugía.

Se evaluó la comorbilidad mediante el índice de Charlson (ích) (categorizándose para nuestro estudio como comorbilidad baja $<=2$ puntos y comorbilidad alta $>=3$ puntos). (1)Para anemia pre operatoria, la transfusión fue indicada en pacientes con valores de hemoglobina menores a $8 \mathrm{~g} / \mathrm{dl}$; y en aquellos pacientes con enfermedad cardiorrespiratoria y/o inestabilidad hemodinámica si los valores de hemoglobina era < $10 \mathrm{~g} / \mathrm{dl}$, según los criterios transfusionales restrictivos marcados por el Comité de Hemoterapia de la institución Para evaluar el estado funcional, se registró 
el grado funcional de la Cruz Roja², de acuerdo a la literatura revisada se desglosa al grado funcional 0,1 y 2 como favorable; y 3,4,5 como desfavorable ${ }^{13}$.

Adicionalmente a la identificación de los factores pronósticos asociados a la mortalidad y morbilidad postoperatoria, se analizó el estado funcional basal y a los 6 meses después de la intervención quirúrgica y finalmente se evaluó la supervivencia y tasa de mortalidad.

El estudio fue aprobado por el Comité de ética Institucional Externo, y el Protocolo fue registrado en la Facultad de Medicina de la Universidad Ricardo Palma y aprobado por la Dirección del Hospital de la F.A.P.

\section{Análisis estadístico}

Se elaboraron tablas de frecuencias para las variables cualitativas, para las variables continuas se obtuvieron la media y la desviación estándar.

Se realizó el análisis bivariado utilizando la prueba de la Chi cuadrado de Pearson para las variables categóricas. En el análisis múltiple se utilizó el modelo de regresión logística. Las variables de resultado fueron el estado funcional basal y a los 6 meses de la intervención quirúrgica, así como la mortalidad. Las variables que resultaron estadísticamente significativas en el análisis bivariado ingresaron a un análisis múltiple para obtener variables independientes. Se calcularon los odds ratios y sus intervalos de confianza al 95\%. Para todos los resultados se considera una significancia $p<0.05$.

El análisis de supervivencia se hizo mediante el método de Kaplan-Meier. Para la comparación de las curvas de supervivencia se realizó mediante el log-rank test.

Para el análisis estadístico se utilizaron el paquete estadístico SPSS para Windows v.23.0.

\section{RESULTADOS}

De los 99 pacientes incluidos se encontró una edad media de 83.51 +- 9,2 años (65-103). El 64,6\% fueron mujeres. El $100 \%$ de los pacientes fueron clasificados ASA II-III. En la tabla 1 se describen las características generales de la población de estudio, considerándose institucionalizado a aquellos pacientes que residen en asilos, albergues, etc. No institucionalizados aquellos que viven en su domicilioparticular.

En nuestro estudio se evidencio que el $24,2 \%$ de los pacientes toman antihipertensivos, por lo que se desprende que la Hipertensión arterial es una enfermedad altamente prevalente en este grupo etario, asi como el $18.5 \%$ consume antidepresivos, 13,16 toma algún AINE y el 20.57 tiene prescripción de otra medicación, se debe tener en cuenta que la polifarmacia es común en los pacientes. Tomando antidepresivos, 45 (18,5\%); ácido acetil salicílico 22 (9,05\%), clopidogrel 15 (6,17\%); antihipertensivos 59 $(24,2 \%)$; hipoglicemiantes 18 (7,4\%); AINES 32 (13,6\%) y otros $50(20,5 \%)$.

Un 53,5\% presentaron al ingreso un íCh superior a 3. El tiempo de demora quirúrgica desde su ingreso fue superior a 48 horas en el 78,8\% de los pacientes.

En el postoperatorio, la presencia de algún evento fue elevada, siendo las cognitivas las más frecuentes, seguidas de los trastornos renales, las cardiovasculares, las complicaciones respiratorias y las infecciones, tomando en cuenta que un paciente puede presentar más de una complicación (tabla 3).

Durante la hospitalización 45,5\% de los pacientes recibió transfusión sanguínea. La mortalidad global fue de $22,5 \%$, la intrahospitalaria encontrada fue del $4,05 \%$, a los 3 meses fue de 11,12\% y a los 6 meses de seguimiento fue de $7,08 \%$. El grado funcional basal fue que 27 (27.3\%) se valen por si mismos, 26 (26,3\%) deambulan con alguna dificultad, 19 (19,2\%) son independientes con bastón, 11 (11,1\%) caminan con andador o requieren poca ayuda de una persona, $10(10.1 \%)$ necesitan ayuda de dos personas o tienen incontinencia habitual, mientras que $6(6,1 \%)$ no caminan, tienen incontinencia total o cuidados de enfermería. Por otro lado; a los 6 meses: 5 (6,49\%) se valen por si mismos, 16 (20.8\%) deambulan con alguna dificultad, 15 (19,5\%) son independientes con bastón, $19(24,68 \%)$ caminan con andador o requieren poca ayuda de una persona, 13 (16,9\%) necesitan ayuda de dos personas o tienen incontinencia habitual y $9(11,7 \%)$ no caminan, tienen incontinencia total o requieren cuidados de enfermería.

En el análisis bivariante de supervivencia, las variables asociadas a mortalidad a los 6 meses fueron: edad avanzada, fracturaprevia, presenciadecomplicaciones, un índice de comorbilidad de Charlson de 3 o más, y presencia de complicaciones respiratorias.

En el análisis multivariado de supervivencia, solo mantuvo su asociación a mortalidad a los 6 meses la variable, fractura previa. Mientras que la edad, una hemoglobina menor a 10, comorbilidad alta y presencia de complicaciones respiratorias, se encontraron asociadas a grado funcional desfavorable.

Se analizó la supervivencia mediante la curva de Kaplan-Meier con y sin fractura previa (figura 3 )

Se observa que aquellos pacientes que presentan fractura previa obtuvieron una mayor mortalidad a diferencia de aquellos que no presentaron este antecedente.

Entonces, se decidió analizar la fractura previa estratificada por sexo (figura 3). 
Tabla 1. Características demográficas, biológicas, clínicas y de tratamiento de los factores asociados a mala evolución en pacientes operados de fractura de cadera mayores de 65 años.

\begin{tabular}{|c|c|}
\hline Variable & $\mathbf{N}=99$ \\
\hline Edad & $83.51+-9,2$ \\
\hline \multicolumn{2}{|l|}{ Sexo } \\
\hline Femenino & $64(64,6 \%)$ \\
\hline Masculino & $35(35,4 \%)$ \\
\hline \multicolumn{2}{|l|}{ Residencia } \\
\hline Institucionalizado con compañía & $14(14,1 \%)$ \\
\hline Institucionalizado sin compañía & $32(32,3 \%)$ \\
\hline No institucionalizado con compañía & $32(32,3 \%)$ \\
\hline No institucionalizado sin compañía & $21(21,3 \%)$ \\
\hline \multicolumn{2}{|l|}{ ASA } \\
\hline I & 0 \\
\hline ॥ & $65(65.7 \%)$ \\
\hline III & $34(34,3 \%)$ \\
\hline IV & 0 \\
\hline \multicolumn{2}{|l|}{ Índice de comorbilidad de Charlson } \\
\hline Ausencia de comorbilidad (0-1) & $3(3 \%)$ \\
\hline Comorbilidad baja (2) & $43(43,4 \%)$ \\
\hline Comorbilidad alta (3 o más) & $53(53,5 \%)$ \\
\hline Hemoglobina al ingreso & $10.1+-1,9$ \\
\hline $\mathrm{Hb}<10 \mathrm{gr} / \mathrm{dl}$ & $57(57,6 \%)$ \\
\hline $\mathrm{Hb}>10 \mathrm{gr} / \mathrm{dl}$ & $36(36,4 \%)$ \\
\hline \multicolumn{2}{|l|}{ Tiempo demora de la cirugía } \\
\hline$>2$ días & $78(78,8 \%)$ \\
\hline$<2$ días & $42(42,4 \%)$ \\
\hline \multicolumn{2}{|l|}{ Técnica quirúrgica } \\
\hline Prótesis total & $20(20,2 \%)$ \\
\hline Prótesis parcial & $17(17,2 \%)$ \\
\hline Placa DHS & $22(22,2 \%)$ \\
\hline Clavo endomedular & $29(29,3 \%)$ \\
\hline Otros & $11(11,1 \%)$ \\
\hline \multicolumn{2}{|l|}{ Transfusión sanguínea } \\
\hline $\mathrm{Si}$ & $45(45,5 \%)$ \\
\hline No & $54(54,4 \%)$ \\
\hline \multicolumn{2}{|l|}{ Tiempo Operatorio } \\
\hline$<45$ minutos & $28(28,3 \%)$ \\
\hline$>45$ minutos & $71(71,7 \%)$ \\
\hline
\end{tabular}

Media \pm desviación estándar; N (\%); mediana

Fuente: Base de datos SPSS v.23. 
Tabla 2. Frecuencia y tipo de complicaciones globales postoperatorias.

\section{Variable}

Cardiocirculatorias

Infarto agudo de miocardio

$3(3,0 \%)$

Arritmias

$12(12,1 \%)$

Edema agudo de pulmón

$4(4,0 \%)$

TEP

$5(5,1 \%)$

$1(1,0 \%)$

AVC

Respiratorias

Hipoxia

$4(4,0 \%)$

Descompensación EPOC

$18(18,2 \%)$

Sobreinfección respiratoria

$4(4,0 \%)$

Otras

$1(1,0 \%)$

Sangrado importante

$15(15,2 \%)$

Renales

Oligoanuria

$14(14,1 \%)$

Insuficiencia renal aguda

$9(9,1 \%)$

Infecciones

De herida

$1(1,0 \%)$

Vías urinarias

$9(9,1 \%)$

Sistémicas

$7(7,1 \%)$

Trastornos cognitivos

Desorientación

$16(16,2 \%)$

Agitación

$1(1,0 \%)$

Reintervención quirúrgica

$7(7,1 \%)$

Otras (complicaciones menores)
$16(16,2 \%)$

Fuente: Base de datos SPSS v.23 N (\%). AVC: accidente vascular cerebral.

EPOC: Enfermedad pulmonar obstructiva crónica. 
Tabla3. Análisis bivariado de factores pronósticos asociados a mala evolución en pacientes operados de fractura de cadera mayores de 65 años.

\begin{tabular}{|c|c|c|c|c|c|c|}
\hline & \multicolumn{3}{|c|}{ Grado de Funcionalidad } & \multicolumn{3}{|c|}{ Mortalidad } \\
\hline \multirow[t]{2}{*}{ Variables } & & Desfavorable & & & Muerto & \\
\hline & OR & IC 95\% & Valor p & OR & IC $95 \%$ & Valor p \\
\hline \multicolumn{7}{|l|}{ Sexo } \\
\hline Masculino & 2.07 & $0.87-4.95$ & 0.100 & 1.57 & $0.61-4.07$ & 0.354 \\
\hline Femenino & 1.00 & & & 1.00 & & \\
\hline \multicolumn{7}{|l|}{ Edad } \\
\hline Mayor de 75 & 7,746 & $1,494-14,23$ & 4,608 & 2.22 & $0.59-8.32$ & 0.236 \\
\hline Menor de 75 & & & & 1.00 & & \\
\hline \multicolumn{7}{|l|}{ Hemoglobina } \\
\hline Menor de 10 & 5,26 & $1,20-3,69$ & 0.022 & 1.20 & $0.66-4.56$ & 0.271 \\
\hline Mayor de 10 & 1.00 & & & 1.00 & & \\
\hline \multicolumn{7}{|l|}{ Fractura Previa } \\
\hline $\mathrm{Si}$ & 1.52 & $0.62-3.70$ & 0.360 & 4.19 & $1.57-11.16$ & 0.004 \\
\hline No & 1.00 & & & 1.00 & & \\
\hline \multicolumn{7}{|l|}{ Comorbilidad } \\
\hline Alta & 6.01 & $2.19-16.60$ & 0.000 & 1.78 & $0.68-4.69$ & 0.244 \\
\hline Baja & 1.00 & & & 1.00 & & \\
\hline \multicolumn{7}{|c|}{ Institucionalidad } \\
\hline $\mathrm{Si}$ & 4.89 & $1.95-12.28$ & 0.001 & 2.14 & $0.83-5.55$ & 0.118 \\
\hline No & 1.00 & & & 1.00 & & \\
\hline \multicolumn{7}{|c|}{ Complicación Respiratorio } \\
\hline Si & 1.983 & $1.38-2.850$ & 0.003 & 2.14 & $0.85-3.62$ & 0.134 \\
\hline No & 0.302 & & & 1.762 & & \\
\hline
\end{tabular}

Fuente: Base de datos SPSS v.23 N (\%). AVC: accidente vascular cerebral.

EPOC: Enfermedad pulmonar obstructiva crónica. 
Tabla 4. Análisis mutivariado para las variables que resultaron significativas con respecto a la variable objetivo Grado Funcional a 6 meses.

\begin{tabular}{|c|c|c|c|c|c|c|c|c|}
\hline \multirow{3}{*}{ Variables } & \multicolumn{5}{|c|}{ Grado de Funcionalidad } & \multicolumn{3}{|c|}{ Mortalidad } \\
\hline & \multicolumn{4}{|c|}{ Desfavorable } & \multicolumn{4}{|c|}{ Muerto } \\
\hline & OR & Potencia & IC $95 \%$ & Valor $\mathbf{p}$ & OR & Potencia & IC $95 \%$ & Valorp \\
\hline \multicolumn{9}{|l|}{ Sexo } \\
\hline Masculino & 2,175 & 0,365 & $0,875-5,87$ & 0,106 & 1,733 & 0,198 & $0,66-4,51$ & 0,264 \\
\hline \multicolumn{9}{|l|}{ Femenino } \\
\hline \multicolumn{9}{|l|}{ Edad } \\
\hline Mayor de 75 & 3,750 & 0,915 & $1,275-11,026$ & 0.016 & 2,075 & 0,222 & $0,552-7,92$ & 0,280 \\
\hline \multicolumn{9}{|l|}{ Menor de 75} \\
\hline \multicolumn{9}{|l|}{ Hemoglobina } \\
\hline Menor de 10 & 6,545 & 0,684 & $1,170-36,6$ & 0,032 & 2,593 & 0,183 & $0,636-10,56$ & 0,184 \\
\hline \multicolumn{9}{|l|}{ Mayor de 10} \\
\hline \multicolumn{9}{|l|}{ Fractura Previa } \\
\hline $\mathrm{Si}$ & 1,183 & 0,504 & $0,429-3,260$ & 0,746 & 3,663 & 0.773 & 1,366- 9,821 & 0.010 \\
\hline \multicolumn{9}{|l|}{ No } \\
\hline \multicolumn{9}{|l|}{ Comorbilidad } \\
\hline Alta & 4,00 & 0.843 & $1,58-10,08$ & 0.003 & 1,949 & 0,212 & $0,709-0,357$ & 0,196 \\
\hline \multicolumn{9}{|l|}{ Baja } \\
\hline \multicolumn{9}{|l|}{ Institucionalidad } \\
\hline Si & 2,200 & 0,862 & $0,894-5,411$ & 0.086 & - & 0,348 & $0,671-4,713$ & 0,247 \\
\hline \multicolumn{9}{|l|}{ No } \\
\hline \multicolumn{9}{|c|}{ Complicación Respiratorio } \\
\hline Si & 6,00 & 0,635 & $1,55-22,94$ & 0.009 & - & 0,317 & & 0,450 \\
\hline No & & & & & & & & \\
\hline
\end{tabular}



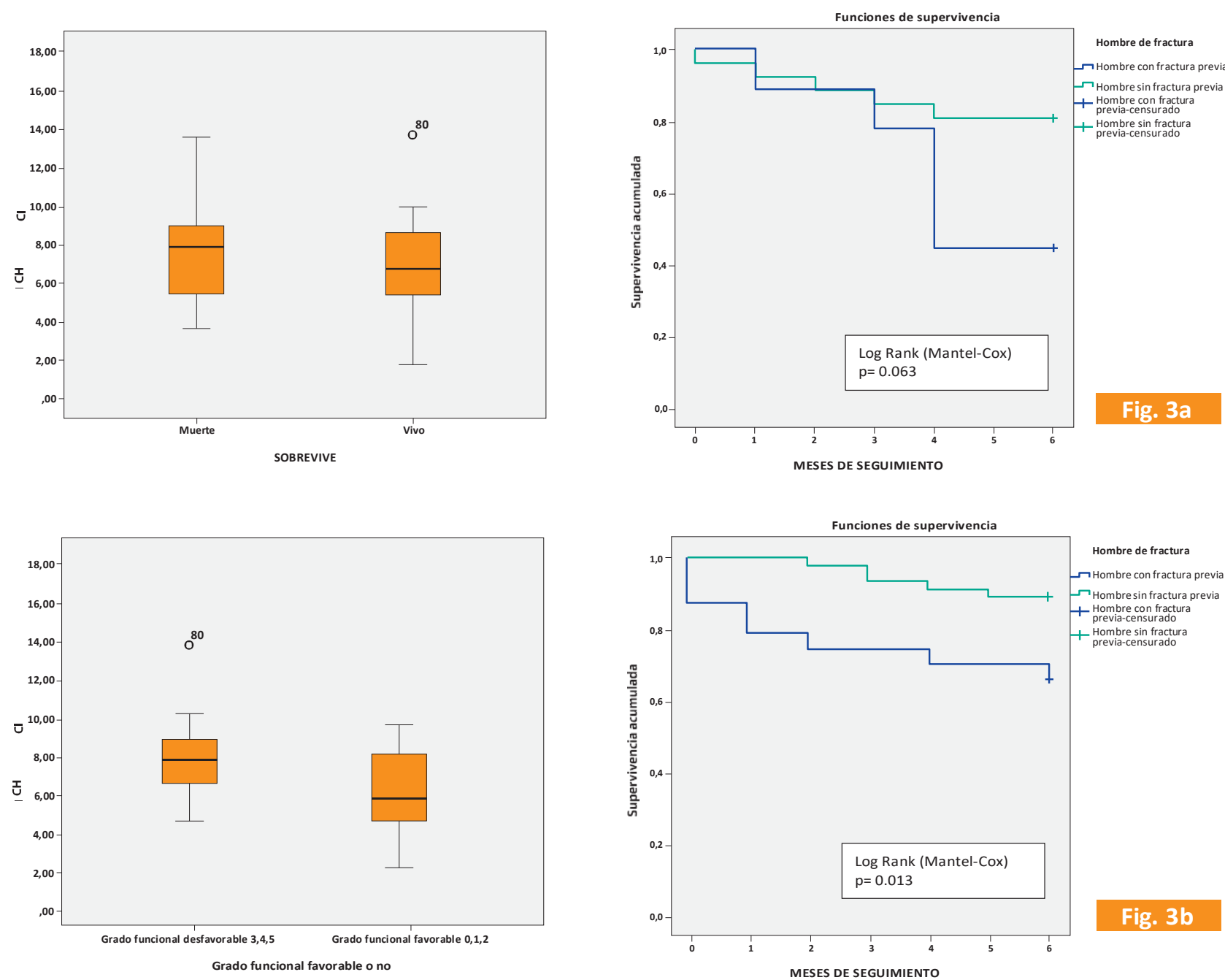

Figura 1. Comparación de supervivencia y grado funcional.

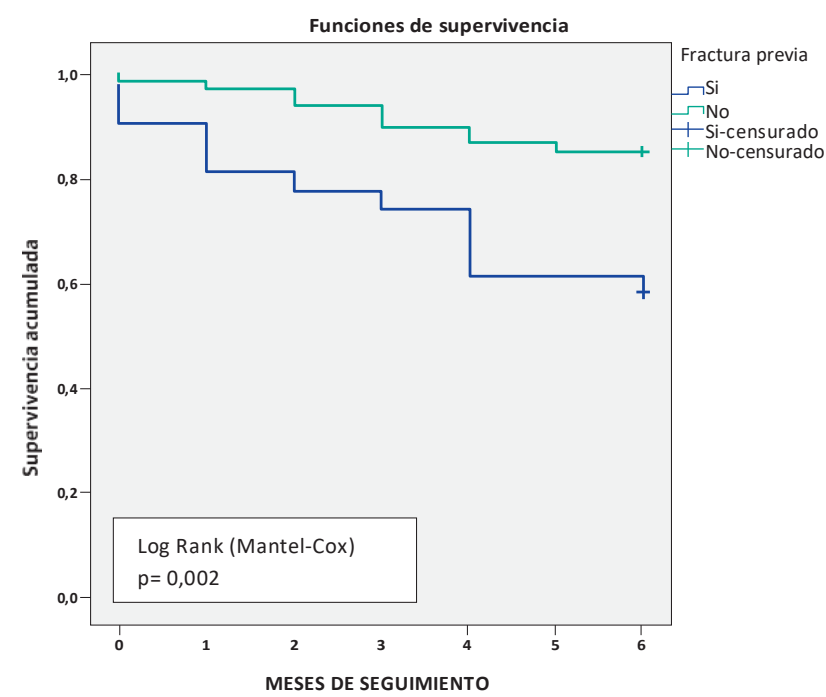

Figura 3. Se analizó la supervivencia en relación a fractura previa por sexo. (Figura 3 a y b).

3a Supervivencia en hombres con y sin fractura previa 3b Supervivencia en mujer con y sin fractura.

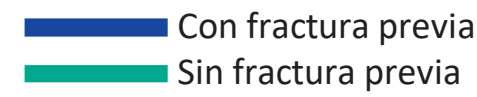

Figura 2. Supervivencia según el antecedente de fractura previa. 


\section{DISCUSIÓN}

En nuestro trabajo, el grupo de población estudiada lo componen pacientes mayores de 65 años divididos en dos grupos con un punto de corte de 75 años, por lo que es de esperar que, tal y como indica la bibliografía consultada, el riesgo de sufrir una fractura de cadera a partir de la quinta década de la vida, se duplica cada cinco años, nuestra población se encontraría ocho veces más en riesgo de presentarla ${ }^{14}$. La edad media que presentaron los pacientes del estudio fue de 83,5 años, ésta cifra se corresponde con la de las series publicadas que incluyen a los pacientes considerados como ancianos ${ }^{15}$.

En relación al sexo, encontramos que el $64,6 \%$ era del género femenino, con una relación mujer/varón 2:1. La incidencia de la fractura de cadera, en nuestro país, es similar a la de otros países del área mediterránea y se sitúa por debajo de los países nórdicos. Es más frecuente en el sexo femenino que en el masculino, con una relación que puede llegar a ser de 3 a 116.

Al evaluar el estatus grado funcional favorable $(0,12)$, cuando comparamos el basal $(72,8 \%)$ con el hallado a los 6 meses (46,73\%), la reducción de grado funcional favorable fue de un $40 \%$; o visto de otro modo la recuperación del grado funcional del $60 \%$. Cuando tomamos globalmentea todos los pacientesy excluimos los dos últimos grados funcionales, obtenemos que un $83,9 \%$ tenían un estado basal en que no necesitaban ayuda para todas las actividades de la vida diaria, y a los 6 meses estos eran de un $71,4 \%$, lo que quiere decir que hubo una recuperación del 73,1\%.

Si se toma en cuenta cada grupo funcional, se observa que de 27 pacientes que previamente eran totalmente independientes, luego de 6 meses del evento solo logran recuperar la independencia total 5 pacientes, en contraste con los grupos funcionales 3 y 4 ( 21 pacientes) que muestran un aumento significativo de pacientes luego de 6 meses de la fractura a 32 pacientes, en comparación con el estudio hecho por Reguant ${ }^{16}$, de 86 pacientes que antes de la fractura tenían una capacidad de marcha normal, un año después de la cirugía solo $33(38,4 \%)$ habían recuperado su capacidad de marcha previa, 47 pacientes $(54,6 \%)$ necesitaban algún tipo de ayuda para caminar, y 6 pacientes (7\%) no caminaban.

En cuanto a residencia de pacientes se encontró que el hecho de estar en una institución o asilo es un factor de riesgo $(\mathrm{OR}=4.89, \mathrm{IC}=1.95-12.28, \mathrm{p}=0,001)$ para un grado funcional desfavorable $(3,4,05$ en la Escala de la Cruz roja), coincidiendo con la literatura ${ }^{17}$.

El estadode salud previode las poblaciones sometidasa estudio es un factor que va a determinar la evolución de unaseriede eventos posibles trasuna fracturade cadera. En general se observa en otros estudios publicados que la cirugía podía retrasarse debido a las pobres condiciones clínicas de los pacientes que aquellos que se operaban antes ${ }^{18}$. En nuestra población de estudio encontramos que el $3 \%$ de los pacientes no presentaron ningún tipo de comorbilidad. El 43,7\% presentó alguna comorbilidad y el $53 \%$ restante fueron tres o más las comorbilidades que se sucedieron. Hemos encontrado la existencia de asociación estadística en la población de estudio en lo que a la referida comorbilidad (OR= $6.01, I C=2.19-16.60, p=0,000$ ), coincidiendo con otras series publicadas ${ }^{16,17}$.

Se obtuvo además que, siendo el paciente institucionalizado, este tiene un mayor riesgo de mala evolución coincidiendo con el estudio realizado por Homero et $\mathrm{al}^{19}$. Pues este tipo de pacientes presenta un mayor riesgo de caídas institucionales. Las caídas a su vez, son la principal causa de accidentes en este grupo de edad. Aunque frecuentemente las caídas no tienen consecuencias mortales, sí afectan la salud y la calidad de vida de las personas ${ }^{20}$.

Por otra parte, los resultados de este estudio muestran que la población intervenida de fractura de cadera en nuestro hospital durante el año 2014 tuvo características similares a otras series publicada ${ }^{8,16,21}$. Nuestros resultados muestran una mortalidad intrahospitalaria de 4,05\% y una mortalidad acumulada a los 3 y 6 meses de $18,20 \%$, cifras inferiores a los publicados por otros grupos $^{17,21,22}$.

No hallamos diferencias significativas en la mortalidad entre los pacientes intervenidos antes o después de las 48 horas del ingreso. Hay mucha controversia en la literatura respecto a la relación tiempo de demora quirúrgica y mortalidad postoperatoria. Diversos autores apoyan que el tratamiento precoz se asocia a una disminución de la mortalidad a corto y mediano plazo. En el estudio realizado en Hospital Cayetano Heredia se encontró que una demora quirúrgica mayor a 48 horas tuvo asociación con complicaciones urinariasy neumonía6. Sin embargo, en nuestro estudio observamos un mayor número de complicaciones a mayor demora quirúrgica alcanzando significancia solo para las complicaciones respiratorias. En el metaanálisis de Shiga et al ${ }^{23}$, la demora quirúrgica superior a $48 \mathrm{~h}$ se asocióa un aumento de la mortalidad a corto y mediano plazo en los pacientes ancianos con fractura de fémur, y la cirugía temprana a un aumento del beneficio en los pacientes de bajo riesgo y en los más jóvenes. Nosotros relacionamos la demora quirúrgica con mortalidad 
a los seis meses, pero a diferencia de Shiga et $\mathrm{al}^{23}$, no hemos encontrado relación entre el tiempo de espera quirúrgico.

Respecto a la hemoglobina como factor pronostico en nuestro estudio se evidencia, una alta asociación con respecto a un grado funcional desfavorable (OR: 5,26; $p=0.022 ; I C=1,20-3,69)$ que coincide con otras series publicadas en las que una anemia severa es un factor asociado a disminución de independencia y con un aumento de mortalidad, sin embargo con respecto a esta última variable nuestros resultados que discrepan con estudios realizados, muy probablemente debido al número de muestra ${ }^{24}$.

Los factores pronóstico relacionados con la mortalidad postoperatoria descritos en la literatura son: la edad avanzada, el sexo masculino, un ASA III y IV, tener 3 o más comorbilidades asociadas al ingreso, la demencia, las complicaciones postoperatorias cardiocirculatorias y respiratorias, y vivir en una residencia o asilo ${ }^{25}$. Nuestros datos después del análisis bivariado coinciden con la literatura en lo que respecta a edad avanzada y tener 3 o más comorbilidades asociadas, pero no en relación al sexo, sin embargo, cuando se compara pacientes con fractura previa según el sexo, existe una mayor supervivencia en pacientes de sexo femenino $(p=$ 0.013)

En el modelo de análisis multivariado, solo continúo siendo factor pronóstico independientes de mortalidad la fractura previa $(p=0.007)$, a diferencia del estudio de Roche et $\mathrm{al}^{25}$, no hemos hallado asociación entre las infecciones respiratorias postoperatorias y la mortalidad. El sexo no influyó, quizá por ser una muestra limitada.

Los resultados obtenidos corroboran la alta morbimortalidad de los pacientes intervenidos de fractura de cadera, las importantes limitaciones en la capacidad de la marcha y el estado funcional de los pacientes a los 6 meses de la cirugía.

En lo que respecta a grado funcional desfavorable, en el modelo multivariado se obtuvo que la edad avanzada, la hemoglobina baja, la alta comorbilidad y las complicaciones respiratorias son factores asociados, lo que constituiría el perfil de un paciente geronte con un mal pronóstico.

\section{RECOMENDACIONES}

Se recomienda realizar estudios prospectivos y de mayor poder estadístico para confirmar la asociación de los factores pronóstico relacionados con las complicaciones postoperatorias, las causas de la demora quirúrgica, la repercusión de la anemia y la demora quirúrgica, y el índice de transfusión; así como poner en consideración el tiempo transcurrido para el inicio de la rehabilitación y la movilización del paciente. Además, es necesario prestar especial atención a aquellos pacientes de edad avanzada y que tienen altas comorbilidades, por medio de una estrategia de estratificación de riesgo según nuestro estudio para así evitar las complicaciones post operatorias que conlleva a la posterior disminución del grado funcional del paciente. Finalmente recomendamos la evaluación geriátrica integral previa a todos los pacientes adultos mayores, a fin de implementar un plan de actuación multidisciplinario previo, de atención individualizada a los pacientes con fractura de cadera antes, durante y posterior a la cirugía, con enfoque a disminuir las complicaciones y la morbimortalidad.

Es por ello que conocer las previsiones sobre la evolución de la fractura de cadera es una cuestión de gran importancia y que excede el ámbito sanitario

\section{CONCLUSIÓN}

Los factores pronósticos para mala evolución en fractura de cadera (grado funcional desfavorable) a los 6 meses fueron: edad, residencia (paciente institucionalizado), alta comorbilidad (Índice de Charslon de más 3 comorbilidades), complicación respiratoria. Finalmente, el antecedente de fractura previa mostro alta asociación a mayor mortalidad de los pacientes a los 6 meses de seguimiento.

Contribuciones de autoría: Los autores participaron en la génesis de la idea, diseño de proyecto, recolección e interpretación de datos, análisis de resultados y preparación del manuscrito del presente trabajo de investigación.

Financiamiento: Autofinanciado.

Conflicto de interés: Los autores declaran no tener conflicto de interés en la publicación de este artículo.

Recibido: 23 de enero del 2019

Aprobado: 30 de julio del 2019

Correspondencia: Renee Flor Vento Benel

Dirección: Avenida Benavides 5440, Surco. Lima, Perú.

Teléfono: 923453756

Correo: ventobnel@hotmail.com 


\section{REFERENCIAS BIBLIOGRÁFICAS}

1. Lin, Xiong D, Peng YQ, Shen ZF. Red Cell Distribution Width as an Independent Predictor of Long-Term Mortality in Hip Fracture Patients: A Prospective Cohort Study. American Society for Bone and Mineral Research. 2015 Junio; 31(1).

2. Report of a scientific group. PREVENTION AND MANAGEMENT of Osteoporosis. WHO Technical Report Series. 2004.

3. CUMMINGS S, RUBIN S, BLACK D. The Future of Hip Fractures in the United States: Numbers, Costs, and Potential Effects of Postmenopausal Estrogen. Clinical Orthopaedics \& Related Research. 1990 Marzo.

4. Chelala Friman R. ¿Será la fractura de cadera la epidemia del siglo XXI? Correo Científico Médico. 2013 Junio; 17(2).

5. ESSALUD. Seguro Social del Perú. [Online]. [cited 2016 Agosto 28 Available from: HYPERLINK "http://www.essalud.gob.pe/" http://www. essalud.gob.pe/.

6. Palomino L, Ramirez R, Julio V, Ray T. Fractura de cadera en el adulto mayor. Acta medica Peruana. 2016 Abril; 33(1).

7. Pages E, Cuxart A. Fracturas de cadera en el anciano: determinantes de mortalidad y capacidad de marcha. Medicina Clinica. 1998; 110.

8. Brauer C, Coca-Perraillon M, Cutler D, Rosen A. Incidence and Mortality of Hip Fractures in the United States. JAMA. 2009 Octubre; 302(24).

9. Fernández-García M, Martínez J, Olmos JM, González-Macías J Hernández JL. Revisión de la incidencia de la fractura en España. Revista de Osteoporosis Metabolismo Mineral. 2015; 7.

10. Brossa Torruella A, Tobías Ferrer J. Demencia y fractura de femur. Revista Española Geriatria yGerontologia. 2007.

11. Hommel A, Ulander K. Influence of optimised treatment of people with hip fracture on time to operation, length of hospital stay, reoperations and mortality within 1 year. Injury. 2008.

12. Cheung AM, Detsky A. Osteoporosis and fractures: missing the bridge? JAMA. 2008;(110)

13. AREOSA SASTRE, AVELLANA ZARAGOZA, BUITRAGO ALONSO CONEJO ALBA. Guía de buena practica clinica en geriatria. Primera ed. Madrid: Rovi; 2007.

14. ALARCÓN ALARCÓN T. Fractura osteoporótica de cadera. ANALES DE MEDICINA INTERNA. 2004 Setiembre;21(2)

15. Marottoli R. Predictors of mortality and institutionalization after hip fracture: the New Haven EPESE cohort. Established Populations for
Epidemiologic Studies of the Elderly. American Journal of Public Health. 1994 noviembre; 84(11).

16. Reguant A. Factores pronóstico de mortalidad en los pacientes mayores con fractura de cadera. 2012 Marzo; 59(. Revista Española de Anestesiología y Reanimación. 2012 Marzo;(59)

17. De la Torre-García M, Hernández-Santana A, Moreno-Moreu N Recuperación funcional tras fractura de cadera en una población anciana, medida con elíndice de Barthel. Revista Española de Cirugía Ortopedicay Traumatologia. 2011 Abril; 55(4).

18. Hoenig $\mathrm{H}$, Rubenstein $\mathrm{L}$, Sloane $\mathrm{G}$. What is the role of timing in the surgical and rehabilitative care of community-oldwelling older persons with acute hip fracture? Archives of Internal Medicine. 1997; 157.

19. Homero G, Marín PP. Caídas en adultos mayores institucionalizados: Descripción y evaluación geriátrica. Revista Medica de Chile. 2003;131(8).

20. Oliver M. Consecuencias médicas: mortalidad y morbilidad de las caídas. Grupo de trabajo de caídas de la Sociedad Española de Geriatría y Gerontología. In Evaluación del anciano con caídas a repetición. Madrid: ditorial Fundación Mapfre Medicina; 1993. p. 3-11.

21. Uribe Ríos A, Castaño Herrera D. Morbilidad y mortalidad en pacientes mayores de 60 años con fractura de cadera en el Hospital Universitario San Vicente Dundación, de Medellín, Colombia. latreia. 2012 octubre; 25.

22. Álvarez Nebreda L. Tratamiento integral de la fractura de cadera en el anciano: modelo predictivo de mortalidad intrahospitalaria. Tesis doctoral. Madris: UNIVERSIDAD COMPLUTENSE DE MADRID, Facultad de Medicina; 2017.

23. Shiga T, Wajima Z, Ohe Y. Is operative delay associated with increased mortality of hip fracture patients? Systematic review, meta-analysis, and meta-regression. Canadian Journal of Anesthesia. 2008 marzo; 55(3).

24. Kovar FM, Endler G, Oswald F. Basal haemoglobin levels as prognostic factor for early death in elderly patients with a hip fracture - A twenty year observation study. Injury. 2015 Enero.

25. Roche J, Sahota O. Effect of comorbidities and postoperative complications on mortality after hip fracture in elderly people: prospective observational cohort study. BMJ. 2005 Diciembre.

26. González González G, López Esqueda F. Correlación del estado funcional y nutricional en pacientes ancianos con fractura de cadera. Medwave. 2012 Junio; XII(5).

27. Lips P. Epidemiology and predictors of fractures associated with osteoporosis. American Journal of Medicine. 1997 Agosto; 18(103).

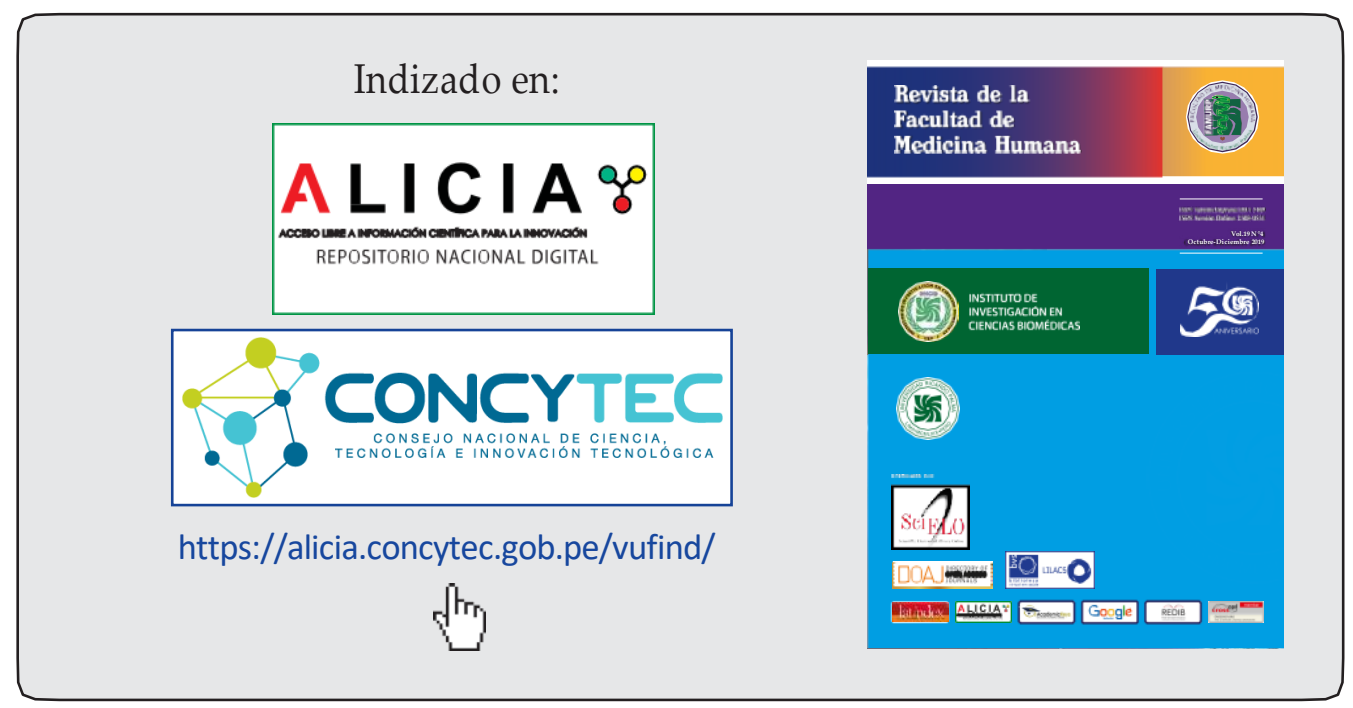

Pág. 94 